\title{
Influence of confinement by smooth and rough walls on particle dynamics in dense hard-sphere suspensions
}

\author{
H. B. Eral, ${ }^{*}$ D. van den Ende, F. Mugele, and M. H. G. Duits \\ Physics of Complex Fluids, IMPACT Institute, University of Twente, P.O. Box 217, 7500 AE Enschede, The Netherlands
}

(Received 14 July 2009; revised manuscript received 8 November 2009; published 14 December 2009)

\begin{abstract}
We used video microscopy and particle tracking to study the dynamics of confined hard-sphere suspensions. Our fluids consisted of 1.1- $\mu \mathrm{m}$-diameter silica spheres suspended at volume fractions of $0.33-0.42$ in waterdimethyl sulfoxide. Suspensions were confined in a quasiparallel geometry between two glass surfaces: a millimeter-sized rough sphere and a smooth flat wall. First, as the separation distance $(H)$ is decreased from 18 to 1 particle diameter, a transition takes place from a subdiffusive behavior (as in bulk) at large $H$, to completely caged particle dynamics at small $H$. These changes are accompanied by a strong decrease in the amplitude of the mean-square displacement (MSD) in the horizontal plane parallel to the confining surfaces. In contrast, the global volume fraction essentially remains constant when $H$ is decreased. Second, measuring the MSD as a function of distance from the confining walls, we found that the MSD is not spatially uniform but smaller close to the walls. This effect is the strongest near the smooth wall where layering takes place. Although confinement also induces local variations in volume fraction, the spatial variations in MSD can be attributed only partially to this effect. The changes in MSD are predominantly a direct effect of the confining surfaces. Hence, both the wall roughness and the separation distance $(H)$ influence the dynamics in confined geometries.
\end{abstract}

DOI: 10.1103/PhysRevE.80.061403

PACS number(s): 82.70.Dd, 64.70.pv, 61.43.Fs, 47.57.J-

\section{INTRODUCTION}

The dynamics of particles in confined colloidal suspensions is an intriguing topic that has raised a lot of attention recently. On one hand, it has been argued that experiments on spatially confined systems can reveal the dynamic length scales that occur in bulk suspensions [1,2] or molecular systems [3]. On the other hand, the influence of confining walls on the mechanisms and time scales of structural rearrangements is also a topic in its own right [4]. Most fluids that have been studied are (near) hard-sphere (HS) suspensions, motivated by their conceptual simplicity at the level of the particle pair interactions. Even for this system, the collective dynamic behavior can already be rather complex.

Bulk HS dynamics has been studied extensively, both at the macroscopic level of the colloidal glass transition $[5,6]$ and at the microscopic level of particle motions [7-9]. On increasing the HS volume fraction, a consistent slowing down of the dynamics was found, which has manifested itself as an increase in the correlation time [10] and a decrease in the diffusion coefficient $[7,11-13]$ or mean-square displacement (MSD) [9,14,15]. However, understanding the mechanisms underlying this slower dynamics beyond qualitative notions can be difficult. From an intuitive point of view, it is clear that the local free volume should play an important role. If this volume is decreased then the motion of individual particles will become more restricted by the (transient) cages formed by the surrounding particles, and consequently the system will slow down. A description of this cage dynamics in terms of length and time scales was given by Weeks and Weitz [16]. However, on approaching the colloidal glass transition, also more collective rearrangements

\footnotetext{
*h.b.eral@utwente.nl
}

have been reported that cannot be covered by a simple cage concept $[3,14]$. This illustrates that our understanding of collective dynamics is still incomplete, even for colloidal hardsphere fluids.

Spatial confinement of a HS suspension will certainly interfere with the mechanisms for structural reorganizations. If dimensions of the fluid container are reduced to the length scales involved in the structural rearrangements, such as those that occur in bulk, then at least certain modes of largescale reorganization will be disabled. For example, while small-scale processes like the caging of individual particles may be sustained until the confinement distance becomes only a few particle diameters, collective motions of, e.g., hydrodynamic clusters will become impossible already at larger confinement distances. Generally, the disabling of dynamic modes is expected to result in a slower dynamics manifested, for example, as a decrease in MSD. Indeed, this trend has also been found in several experimental $[1,2]$ and simulation [17-19] studies. However, a deeper insight into how the observed reduction in MSD comes about is still lacking. Several shortcomings can be pointed out:

(1) The effects of the particle-wall interaction are still incompletely understood. The simplest case is that of hard spheres confined by hard walls: here, only the roughness of the wall has to be considered. In several computer simulation studies, the wall roughness has been found to strongly affect the local particle dynamics $[4,18,20]$. However, in experiments [1] the permanent adhesion of a fraction of the particles to a smooth wall did not have a noticeable effect. In another experimental study [2], a layer of particles was deposited and subsequently sintered in order to roughen the wall. As far as we know, no other experimental studies exist that specifically address the effect of the wall roughness on particle dynamics in confined fluids.

(2) How the effects of the confining walls on the particle dynamics are transmitted from the walls into the suspension 

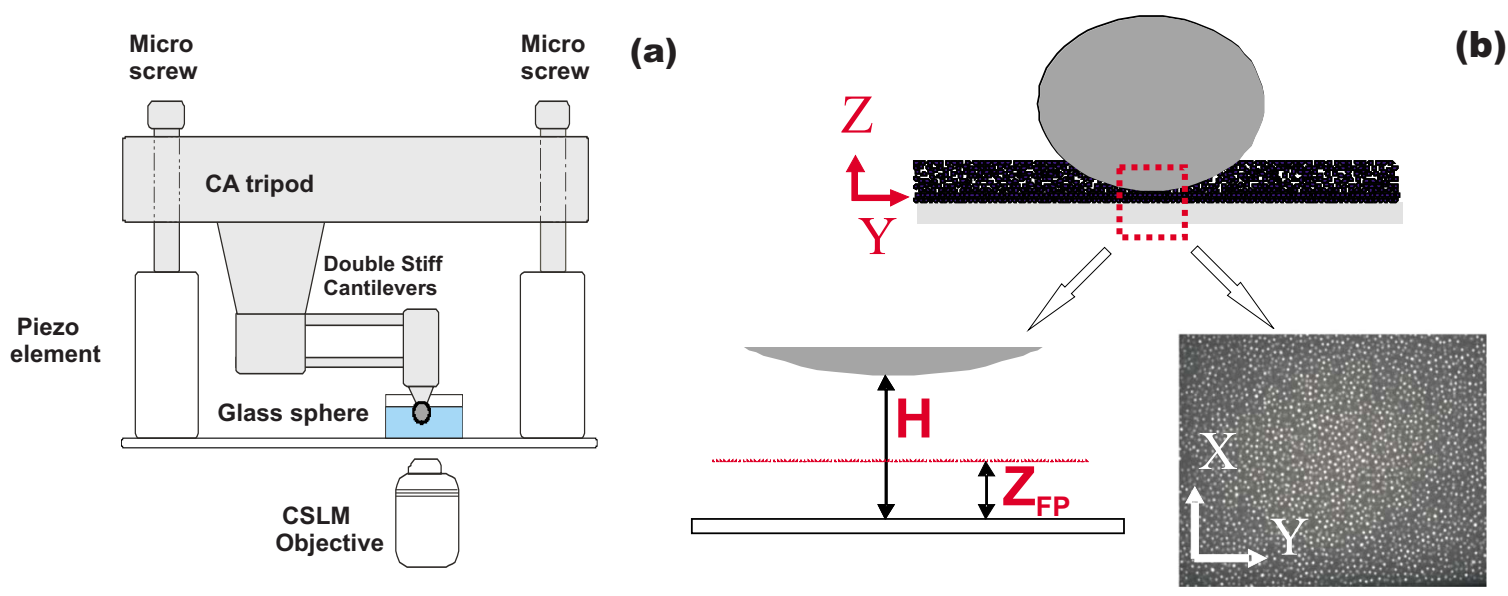

FIG. 1. (Color online) (a) Schematic illustration of our CA. Colloidal fluid is confined between a glass plate and a sphere in a quasiparallel geometry. The position of the sphere can be accurately controlled via the piezo stage on which the CA tripod is resting. Observations of the particles are made from below using a CSLM. (b) Close-up of the sphere-plane geometry with confinement gap $(H)$ and height of the focal plane $\left(Z_{F P}\right)$. Also a typical CSLM image of a confined fluid is shown (scale: $\left.87 \times 66 \mu \mathrm{m}^{2}\right)$.

is also an unsettled issue. Depending on the restructuring mechanism, the particle dynamics could be collective or alternatively very local and dependent on the separations from the walls. From the literature it is not clear which cases to expect. In computer simulations on Lennard-Jones fluids, a confinement-induced glass transition was reported as a collective effect of all layers [4]. Another simulation study [18] reported a lack of evidence that the particle layer closest to the wall was decoupling dynamically from the rest. However, in simulations on HS confined by spherical cavities with smooth and rough walls, Nemeth and Lowen [21] found MSDs that depend strongly on the distance from the wall. For the smooth walls, the tangential MSDs were larger at the wall as compared to the interior, whereas for rough walls a different spatial dependence was found. Experiments on HS suspensions confined between two flat plates, performed by Nugent et al. [1] showed yet again different trends: MSDs in planes parallel to the wall did not depend on the distance from the wall. If and how these different findings could be reconciled is not clear at present, and hence a further study is warranted.

(3) To what extent local variations in the MSD of confined fluids could be attributed to local differences in volume fraction is also unknown at present. The issue has been raised, but only addressed in a few studies. Mittal et al. [17] studied this issue via computer simulations and found that higher local densities in a confined HS fluid correlated with a faster local diffusion. This counterintuitive result was explained via the increased Widom insertion probability. Also experimental data that allow correlating between local MSD and local volume fraction are scarce. Dullens and Kegel [22] studied HS suspensions at a single wall and found, up to a volume fraction $\varphi=0.54$, a continuous decrease in MSD. Sarangapani and Zhu [2] measured local volume fractions in two confined samples but did not aim for correlation with local MSDs. Nugent et al. [1] measured MSDs and local densities and found that one of the two particle species of their bidisperse system showed a concentration peak at the wall, but no change in the MSD parallel to the wall.
In this paper, we shed light on the three mentioned issues via an experimental study on confined hard-sphere suspensions, focusing our analysis on the MSDs measured with video particle tracking. A systematic study was performed into the effect of confining a colloidal fluid by two different walls (smooth and rough), on local particle dynamics. We found that progressive confinement caused a dramatic decrease in the MSD, without significant changes in the overall concentration. Local concentration variations within the confinement gap did occur, however, and showed significant asymmetry when comparing the different walls. Also the MSDs were significantly different at the smooth and rough walls. These trends will be analyzed, from which it will be concluded that the roughness of the wall and the distances from the rough and smooth walls have a much stronger influence on MSD than the variations in local volume fraction.

\section{EXPERIMENTAL METHODS}

\section{A. Confinement apparatus}

Our homemade confinement apparatus (CA) [23] is sketched in Fig. 1. Colloidal suspensions are confined between two glass surfaces (a sphere and a cover slide). The sphere $\left(D_{\text {sphere }}: 2 \mathrm{~mm}\right.$, Duke Scientific) is glued onto a holder, which is connected to the CA tripod via stiff double cantilevers. The tripod rests on a piezo stage via three microscrews. Coarse control over the gap height $H$ is achieved via the screws and guided by a visualization using fluorescent liquid. Fine control is achieved using a computer driven piezo stage (Physik Instrumente) with a vertical range of $20 \mu \mathrm{m}$ and an accuracy of $0.01 \mu \mathrm{m}$. In typical measurements, $D_{\text {sphere }} \gg H$, which means that an effective planeplane geometry is obtained, independent of the precise alignment. Both confining surfaces were analyzed for topography with atomic force microscopy (AFM). The results are shown in Fig. 2. The root-mean-square (rms) value of the height variations reveals that, while the bottom surface is smooth $(\mathrm{rms}=10 \mathrm{~nm})$, the surface of the glass sphere shows significant roughness $(\mathrm{rms}=0.13 \mu \mathrm{m})$. 


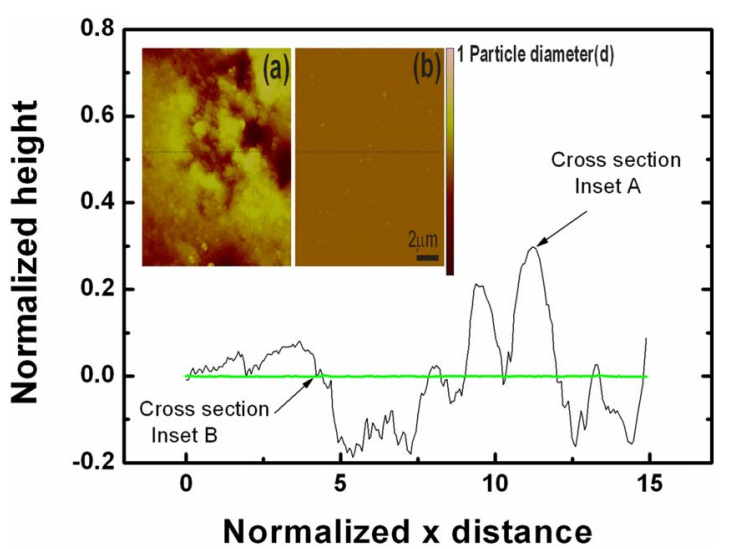

FIG. 2. (Color online) Representative cross sections of AFM topography analysis of the two confining surfaces. Axis ranges are scaled to the diameter $d$ of the colloidal particles. Inset (a) shows topography image of the rough glass sphere while inset (b) shows the smooth confining wall, i.e., cover slip. The color bar of both insets shows the height, also scaled to $d$. The dotted lines correspond to the line profiles in the main graph.

\section{B. Colloidal fluid}

Core-shell silica particles with outer diameter $d$ $=1.1 \mu \mathrm{m}$ were synthesized following the method described in [24], which entails the deposition of nonfluorescent silica onto a fluorescent core. In our case, the core contains Rhodamine isothiocyanate and had a diameter of $\approx 500 \mathrm{~nm}$. Such core-shell particles allow for an accurate localization of particle centers from video microscopy images, even at high volume fractions. The polydispersity was assessed to be $8 \%$ from scanning electron microscopy images (see supplementary material [25]). To obtain (near) hard-sphere suspensions, the solvent was changed to a refractive index matching mixture of $\mathrm{H}_{2} \mathrm{O} / \mathrm{DMSO}$ [26] to minimize the van der Waals attractions (and to optimize visualization, see Sec. II C), while $\mathrm{LiCl}$ was added to a final concentration of $0.01 \mathrm{M}$, to reduce the electrical double layer thickness to $\approx 7 \mathrm{~nm}$.

Soft centrifugation (1000 g for $1 \mathrm{~h}$ ) was used to concentrate the fluid. This was done directly in the sample holder, which was made of an open cylindrical tube (diameter: 20 $\mathrm{mm}$ ) glued onto a round glass cover slip. Sediments were prepared at volumes ranging in between 0.25 and $1.5 \mathrm{ml}$, at an initial volume fraction $\varphi \approx 0.66$, in line with expectations for random close packing of a system with $8 \%$ polydispersity [27]. After the removal of the supernatant the tube was weighed, and the amount of solvent needed to achieve the target $\varphi$ was added. Then the sediment was resuspended using a whirl mixer. Samples were prepared at $\varphi$ values ranging from 0.15 to 0.57 .

The precise volume fractions were ascertained $a$ posteriori from the confocal scanning laser microscope (CSLM) observations. Localizing all particles (see Sec. II C) allowed us to obtain $\varphi$ values as follows: $(X, Y, Z)$ control volumes $(V)$ were defined as $(65 \mu \mathrm{m} \times 65 \mu \mathrm{m} \times \Delta Z)$ with either $\Delta Z=H$ (for the global volume fraction) or $\Delta Z \ll H$ (for local $\varphi$ values). Then it was calculated for each particle $(i)$, which fraction $\left(f_{i}\right)$ of its volume fell within the control volume $V$, by using the relative location of the particle to the boundaries of $V$ and assuming $d=1.12 \mu \mathrm{m}$. For $V \gg \pi d^{3} / 6$ this mostly corresponded to $f_{i}=1$, but for small $\Delta Z$ mostly $0 \leq f_{i}<1$. Then taking $N_{e f f}=\Sigma N_{i} f_{i}$ as the effective number of particles allowed us to calculate $\varphi$ as $N_{e f f} \pi d^{3} / 6 V$. In this way it was ensured that $\varphi$ always represents a physical volume fraction, even for $\Delta Z<d$. Typical values were $N_{e f f} \gg 1000$ (global $\varphi$ ) and $N_{e f f} \approx 1000$ (local $\varphi$ ). In the case of local volume fractions it must be underlined that this is a strictly geometrical definition; hence, it will be designated as $\varphi_{s}$ from now on.

An accurate and consistent way to measure $d$ was to analyze a CSLM image of a very dense layer on a cover slip with many particles touching each other. The average distance between touching particles was found to be $1.12 \pm 0.03 \mu \mathrm{m}$, giving an estimated relative inaccuracy in the volume fraction of $8 \%$. Also the magnitude of $H$ was measured more accurately, by making use of the CSLM recordings. The location of the bottom surface was defined as $0.5 d$ below the measured average $Z$ position of all particle centers in the lowest layer. The top surface was localized by extrapolating the steep flank of the concentration profile to zero (see Fig. 6) and adding $0.5 d$. Considering the particle polydispersity and the rms roughness of the top surface, we estimate the inaccuracy of $H$ to be $0.2-0.3 \mu \mathrm{m}$.

In the presence of confining surfaces, concentrated suspensions may change their structures and dynamics. However, the time that is needed for such changes is not known $a$ priori and may depend on the volume fraction. For this reason, we studied confined systems at $\varphi=0.33-0.42$, i.e., well below the glass transition point for hard spheres at $\varphi=0.58$ [5]. Moreover, we also examined the influence of the waiting time after the fluid had been confined to a new gap height. Here, it turned out that waiting $4 \mathrm{~h}$ instead of the (standard) $1 \mathrm{~h}$ gave similar results. Furthermore, we checked whether the colloidal fluid crystallized at time scales comparable to the duration of our experiment. No evidence of crystallization was found, in accordance with the $8 \%$ polydispersity and a previous study [28].

\section{Confocal microscopy and particle tracking}

The CSLM was an UltraView LCI10 system (PerkinElmer) containing a Nikon Eclipse inverted microscope equipped with a Nipkow disk (Yokogawa module) and $100 \times$ numerical aperture 1.3/oil objective. When tracking particle dynamics, we imaged horizontal $(X, Y)$ focal planes for $240 \mathrm{~s}$ at a rate of 10 frames/s. For measuring particle locations in three dimensions (3D) we measured series of images along the $Z$ direction (up to $30 \mu \mathrm{m}$ from the bottom, taking $\approx 60 \mathrm{~s}$ ) at $0.1 \mu \mathrm{m} / \mathrm{step}$. The images were processed via the available particle tracking codes in two dimensions and 3D [29]. The accuracy of locating the centroid of particles in 3D was $0.02 \mu \mathrm{m}$ in $X-Y$ and $0.05 \mu \mathrm{m}$ in $Z$ direction. The $Z$ resolution of our two-dimensional particle tracking was measured to be $\approx 0.8 \mu \mathrm{m}$ (full width at half maximum), by localizing (almost stationary) particles in a three-dimensional volume and subsequently analyzing per focal plane, up to which distance from the focal plane particles were still accepted by the two-dimensional particle tracking code (using typical selection criteria for brightness, object size, etc.). 


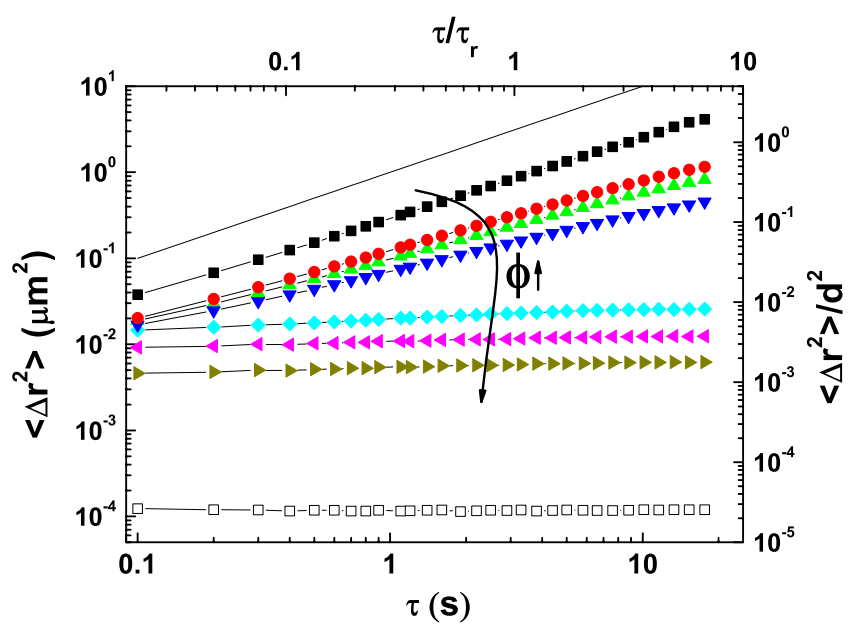

FIG. 3. (Color online) MSD vs time lag for bulk samples at different volume fractions. Lower abscissa and left ordinate: lag time $(s)$ and mean-square displacement $\left(\mu \mathrm{m}^{2}\right)$. Upper abscissa and right ordinate: normalized data, where $\left\langle\Delta r^{2}(\tau)\right\rangle$ was divided by $d^{2}$ $=1.32 \mu \mathrm{m}^{2}$ and $\tau$ by the Brownian time $\left(\tau_{r}\right)$, where $\tau_{r}=d^{2} / 4 D_{0}$ equals $3.54 \mathrm{~s}$. The symbols indicate volume fractions $(\varphi)$ of 0.16 $(\boldsymbol{\square}), 0.26(\bullet), 0.37(\boldsymbol{\Delta}), 0.42(\boldsymbol{\nabla}), 0.52(\bullet), 0.54(\boldsymbol{\bullet})$, and 0.57 $(\boldsymbol{\nabla})$. Open symbols $(\square)$ indicate the noise floor.

Image-time series aimed at studying particle dynamics typically consisted of 2500 time steps. After localizing all particles in each frame and building trajectories, MSDs in the horizontal plane were calculated using

$$
\left\langle\Delta r^{2}(\tau)\right\rangle=\left\langle\left\{[x(\tau+t)-x(t)]^{2}+[y(\tau+t)-y(t)]^{2}\right\}\right\rangle,
$$

where $x(t)$ is the $X$ position of a particle at real time $t, \tau$ is the lag time, the braces \{\} indicate an averaging over all times $t$, and the angular brackets \langle\rangle indicate an averaging over all particles. In the calculation of $x(t)$ and $y(t)$ a correction for mechanical drift was applied by subtracting the measured average displacement of the ensemble of particles. The typical accuracy in the MSD was $1.0 \times 10^{-4} \mu \mathrm{m}^{2}$. In the following, MSDs will be fitted as

$$
\left\langle\Delta r^{2}(\tau)\right\rangle=A\left(\tau / \tau_{0}\right)^{\alpha}
$$

with $A$ as the amplitude, $\tau_{0}$ as the shortest exposure time, and $\alpha$ as the exponent indicating the behavior of Brownian motion ( $\alpha=1$ diffusive, $\alpha<1$ subdiffusive). The fitting range for $\alpha$ was taken from 0.1 to $10 \mathrm{~s}$.

\section{RESULTS}

\section{A. Particle dynamics in bulk fluids}

As a reference, we present in Fig. 3 a series of MSD curves measured for the bulk suspensions at different volume fractions $\varphi=0.16-0.57$. To ensure that the measurements pertained to the dynamic behavior in bulk, observations were done at $Z_{F P}=30 \mu \mathrm{m}$, which will be shown to be sufficiently far away from the wall. As $\varphi$ is increased both the amplitude $A$ and the exponent $\alpha$ of $\left\langle\Delta r^{2}(\tau)\right\rangle$ [see Eq. (2)] become smaller. The exponent $(\alpha)$ is observed to change from almost 1.0 (as for a viscous liquid) to 0.0 (as for a solid). The oc- currence of a plateau for $\varphi \geq 0.52$ suggests a solidlike microscopic dynamics already starting at this volume fraction, whereas the macroscopic glass transition for colloidal HS is supposed to occur at $\varphi=0.58$ [5]. These results are qualitatively in agreement with other studies and will be further analyzed in Sec. IV A.

\section{B. Particle dynamics in confined fluids}

The effect of confinement on the MSD was examined for bulk volume fractions of $0.33,0.38$, and 0.42 , in the range where the bulk fluid still showed liquidlike behavior. For all three volume fractions the trends were similar; results will be presented for the system at $\varphi=0.33$, unless mentioned otherwise. Figure 4(a) shows the MSDs taken in the midplane of the gap $\left(Z_{F P}=H / 2\right)$ between plate and sphere. On decreasing $H$ from $20 \mu \mathrm{m}$ to 1 particle diameter, strong changes in the MSD curve are observed: a reduction in amplitude as well as a decrease in the exponent, eventually reaching zero. These changes are qualitatively similar to the effects of increasing the volume fraction in bulk systems, and that eventually led the system into the glass state (Fig. 3). Same trend is observed for Fig. 4(b) where the MSDs at a fixed distance $(2 \mu \mathrm{m})$ away from the rough and smooth walls are monitored.

A systematic study was performed, covering MSDs as a function of distance from walls. It then turned out that the MSDs of our confined fluids are not constant over the gap. This is demonstrated by the comparison of Fig. 4(a), which shows the MSDs measured at the midplane, with Fig. 4(b) in which the MSDs measured at $Z_{F P}=2 \mu \mathrm{m}$ and $Z_{F P}=H$ $-2 \mu \mathrm{m}$ are plotted. Clearly, near the surfaces the MSDs are different from those at the midplane. It is also evident from Fig. 4(b) that near the smooth bottom plate, the MSDs are consistently lower than at the rough surface of the top sphere. This difference will be further discussed in Sec. IV B.

We examine the $\operatorname{MSD}\left(H, Z_{F P}, \tau\right)$ function in more detail by plotting the magnitude of $\left\langle\Delta r^{2}(\tau)\right\rangle$ evaluated at a lag time $\tau=10 \mathrm{~s}$, for many $\left(H, Z_{F P}\right)$ combinations. At this lag time, the differences between the MSDs are more pronounced than at shorter times, while the typical errors found at long times (due to poor statistics and gradients in drift $[30,31,35]$ ) are still small.

The solid symbols in Fig. 5 show the spatially resolved MSDs for the same gap heights as in Fig. 4(a) (including an additional experiment for which $H \rightarrow \infty$ ). It is observed that the MSDs show a maximum in between the two confining surfaces. This maximum is not in the middle of the gap but closer to the surface of the top sphere. It is also noted that the $Z_{F P}$ dependence of the MSD is gradual. Although the MSDs become rather small due to the confinement, they all remain well above the noise floor, indicating that the particles are still rearranging themselves. This finding is corroborated by our trajectory analysis (not shown), in which all particles still show motion. Hence, we found no evidence that particles had permanently stuck to any surface.

Considering the possibility of layer formation at walls and the clear dependence of the MSD on the concentration in 

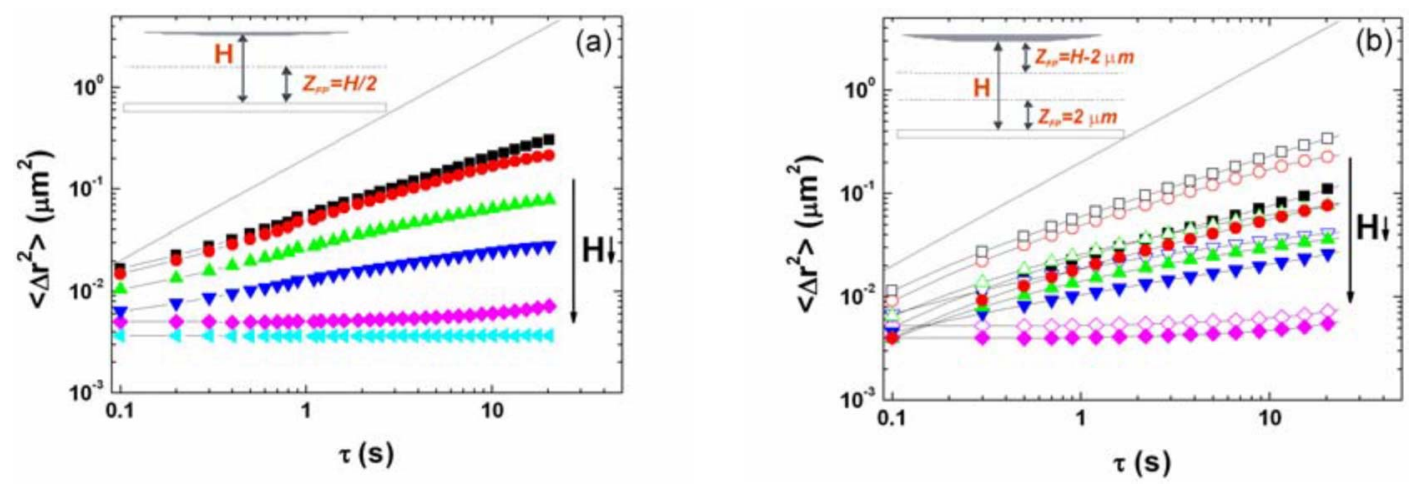

FIG. 4. (Color online) MSD vs time lag plot for decreasing confinement gap $(H)$. In (a), each curve indicates the MSD at the midplane $Z_{F P}=H / 2$ (see Fig. 1). Correspondence between $H(\mu \mathrm{m})$ and symbols: $20(\boldsymbol{\square}), 16(\bullet), 12(\boldsymbol{\Delta}), 8(\boldsymbol{\nabla}), 4(\bullet)$, and $\approx 1.3(\bullet) \mu \mathrm{m}$. (b) MSD vs time lag for different gap heights $H$, now for planes $2 \mu \mathrm{m}$ away from the confining walls. Open and closed symbols correspond to rough (top) and smooth (bottom) walls, respectively. Volume fraction $(\varphi)$ of the sample is 0.33 . Qualitatively similar behavior was observed for samples at $\varphi=0.38,0.42$.

bulk HS systems (Figs. 3 and 7), the question arises to what extent the variations in MSD could be attributed to local volume fraction effects. The main graph of Fig. 6 shows the "geometrical local volume fraction" profile $\varphi_{\mathrm{s}}(Z)$, calculated as explained in Sec. II B. Clearly, the profiles are strongly peaked near the bottom wall. The peaks are approximately equally spaced (distance $\Delta Z_{p p}: 1.14 \mu \mathrm{m}$ ) and their amplitude becomes progressively smaller as the distance from the wall is increased. This is similar to the behavior that was found in previous studies on layering of HS suspensions [32-34]. In our case the depth of the first minimum is remarkably low, corresponding to a very low occurrence of sphere centers (as was checked from the three-dimensional localization data). Furthermore, the first peak to peak dis-

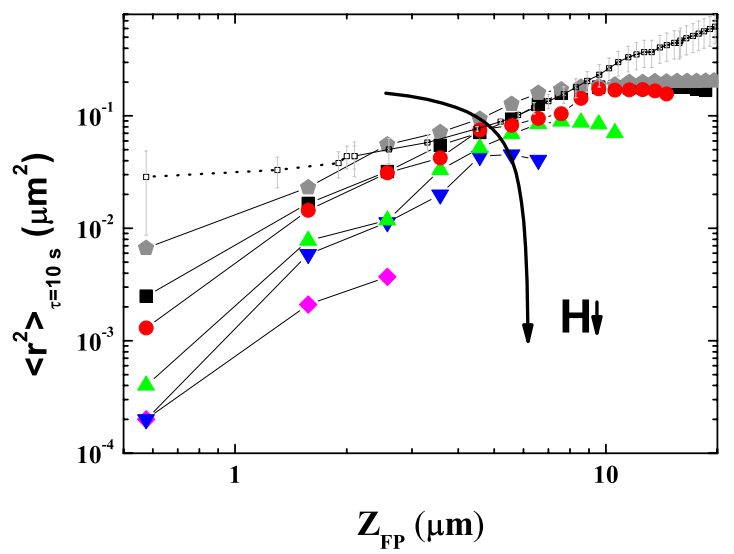

FIG. 5. (Color online) Spatially resolved behavior of $\mathrm{MSD}_{\tau=10 \mathrm{~s}}$ for the fluid at $\varphi=0.33$, confined at different gap heights $H$. Correspondence between $H(\mu \mathrm{m})$ and symbols: $20(\mathbf{\square}), 16(\boldsymbol{\bullet}), 12(\mathbf{\Delta})$, $8(\boldsymbol{\nabla})$, and $4(\boldsymbol{)})$. The symbol belongs to the experiment where the second confining surface is far away $(H \rightarrow \infty)$. Corresponding open symbols indicate "bulk" MSD values calculated from the local volume fraction showing what $\mathrm{MSD}_{\tau=10 \mathrm{~s}}$ would be if the system was bulk and dynamics were solely governed by volume fraction. The open symbols have been calculated from the linear fit to a characteristic curve in the inset of Fig. 6. The error bars have been calculated from different fits to inset of Fig. 6. The dotted part of open symbols indicates extrapolation. See text for further details. tance is slightly larger; we offer no tentative explanation for this.

Confinement by the second surface causes only little changes in the $\varphi_{\mathrm{s}}(Z)$ profile near the bottom surface; the strongly peaked structure remains and also the $\varphi_{\mathrm{s}}$ values superimpose fairly well. The overall volume fraction shows a small decrease upon confining the fluid from $H \rightarrow \infty$ to $H$ $=20 \mu \mathrm{m}$, but upon progressive confinement it shows less than $5 \%$ relative variation. Also the concentration profile under the top sphere can now be studied. Although some layering occurs, the peaks are by far less pronounced than at the bottom surface. Again this points at an important difference between the top and bottom surfaces, which will be discussed in Sec. IV B.

We return to the question whether the variations in MSD could be due to the variations in local volume fraction only. We examine this by measuring the relation between the local

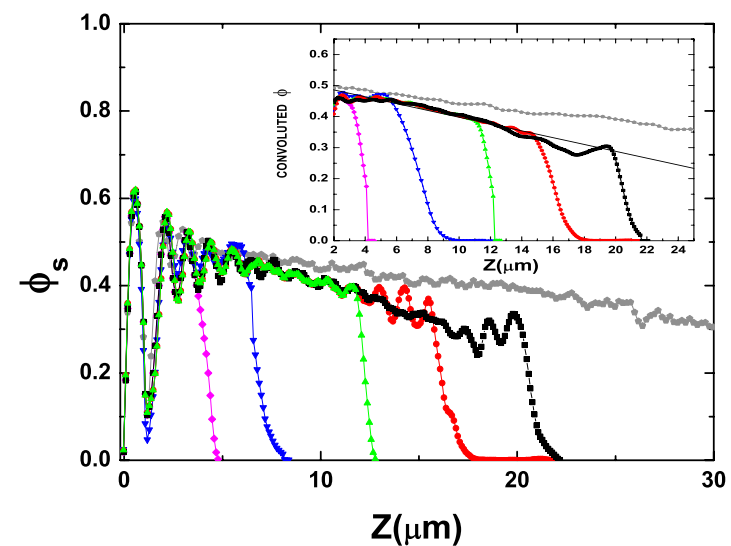

FIG. 6. (Color online) Geometric volume fraction $\left(\varphi_{s}\right)$ vs $Z$ for different confinement gaps: correspondence between $H(\mu \mathrm{m})$ and symbols: $20(\boldsymbol{\square}), 16(\mathbf{\bullet}), 12(\boldsymbol{\Delta}), 8(\boldsymbol{\nabla})$, and $4(\bullet)$. The symbol belongs to the experiment where the second confining surface is far away $(H \rightarrow \infty)$. $\varphi$ indicates local volume fraction calculated in a bin. Inset: convoluted volume fraction $(\varphi)$ vs $Z$ histogram, as needed for generating reference $\mathrm{MSD}_{\tau=10 \mathrm{~s}}$ data at in Fig. 5. Solid line is linear interpolation to convoluted volume profile used to calculate expected MSD values in Fig. 5. See text for further details. 
$\varphi$ and the local MSD and subsequently comparing each local MSD magnitude with the value that it would have in a bulk system at the local $\varphi$. A question that then arises is down to which length scale a calculated local $\varphi$ would still be meaningful. Since the MSD of a particle should at least depend on the cage defined by its direct neighbors, the use of $\varphi$ values calculated at smaller length scales than this cage length would not make sense (the precise length scale at which local $\varphi$ does matter still has to be assessed for confined systems). To take this aspect into account and sample the $\varphi(Z)$ profile at a more appropriate length scale, we convoluted the $Z$ histogram of particle center locations with a block profile of width $\Delta Z_{p p} \approx d$. Subsequently we applied an additional convolution with a Gaussian kernel with $0.8 \mu \mathrm{m}$ FWHM to account for the optical $Z$ resolution of the MSD measurement (see Sec. II C). These operations produce the $\varphi(Z)$ profiles shown in the inset of Fig. 6. Looking up the $\varphi$ values from this profile and interpolating against the $\operatorname{MSD}(\varphi)$ relation for bulk samples then produces the densely dotted $\operatorname{MSD}(Z)$ data shown in Fig. 5. Since slightly different convolutions and interpolations would have been possible, we represent the effect thereof on the MSD values by error bars. Now comparing the interpolated MSD curves to the measured data, it becomes clear that the data interpolated from the $\operatorname{MSD}(\varphi)$ relation strongly overestimate the MSDs of the confined systems, and the more so as $H$ gets smaller. This demonstrates that the dramatic decrease in MSD near the surface(s) can only for a very small part be attributed to variations in local volume fraction.

Confinement experiments were done at several volume fractions $(\varphi=0.33,0.38,0.42)$, but so far only the results at $\varphi=0.33$ were shown. Importantly, for all three samples a similar behavior was found when the gap height was reduced from (effectively) infinite to just a few particle diameters. To illustrate this, we plotted MSD data at $\tau=10 \mathrm{~s}$, for both bulk and confined fluids in Fig. 7. For all three confined fluids, the local volume fraction changes only a little with confinement, and dramatic reductions in MSD compared to the bulk are found. At the smallest gaps, it seems that the volume fraction differences even do not matter anymore. This strong dependence of the MSD on $H$ as compared to the dependence on $\varphi$ is also illustrated in the inset of Fig. 7. This underlines once more that the reductions in MSD are largely due to confinement, rather than due to the local volume fraction.

\section{DISCUSSION}

\section{A. Comparison with hard-sphere systems}

Besides using the MSDs in Fig. 3 for comparison between bulk and confined fluids, it is also interesting to examine if they show the behavior as expected for hard spheres. From the curve at $\varphi=0.16$ an apparent viscosity of $6.0 \mathrm{mPa} s$ was extracted using the Stokes-Einstein equation; this gives a relative viscosity of 1.47 , which is fairly close to $\approx 1.6$ as expected for HS $[36,37]$. However, the calculation of a viscosity from MSD data using the generalized Stokes-Einstein relation $[38,39]$ is not trivial for nondilute HS suspensions. Alternatively also the normalized diffusion coefficient $D / D_{0}$, with $D_{0}$ as the diffusion coefficient for $\varphi \rightarrow 0$, can be com-

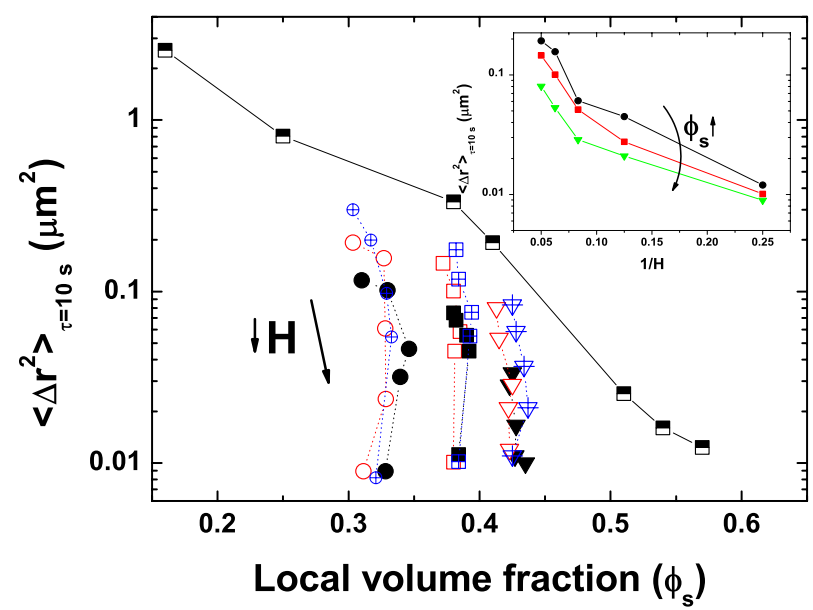

FIG. 7. (Color online) $\left\langle r^{2}\right\rangle_{\tau=10 s}$ vs local volume fraction. Half full squares indicate bulk measurements; all other symbols refer to confinement. Open red: $Z_{F P}=H / 2$, solid black: $Z_{F P}=2 \mu \mathrm{m}$, and crossed blue: $Z_{F P}=H-2 \mu \mathrm{m}$. Symbol shapes indicate the initial volume fraction: $\varphi=0.33(\bullet), 0.38(\boldsymbol{\square})$, and $0.42(\boldsymbol{\nabla})$. $H$ varies between 20 and $4 \mu \mathrm{m}$ for this graph. Inset: same MSD data at $Z_{F P}=H / 2$ plotted vs $1 / H$.

pared to literature. For $\varphi \approx 0.16$, Ottewill and Williams [40] found a normalized self-diffusion coefficient of $\approx 0.72$ at short times and $\approx 0.44$ at long times. This suggests that our data at $\varphi=0.16$ (where $D / D_{0}=0.68$ ) show the short-time self-diffusion. The subdiffusive behaviors $(0<\alpha<1)$ at higher volume fractions then show intermediate regimes between short- and long-time self-diffusion.

Also direct comparisons with MSDs of other HS systems are possible. Kasper et al. [28] studied cross-linked poly-t-butylacrylate particles $(d=910 \mathrm{~nm})$ in 4-fluorotoluene at $Z_{F P} \approx 10 \mu \mathrm{m}$ (or 11 particle diameters) for $\varphi$ $=0.32-0.60$. They also observe a transition from an almost diffusive behavior (at $\varphi=0.32$ ) to a plateau (at $\varphi=0.60$ ), with subdiffusive behaviors $(0<\alpha<1)$ in between. After normalizing MSDs as $\left(\Delta r^{2}\right) / d^{2}$ and $4 \tau D_{0} / d^{2}$, which should result in a master curve for hard spheres, their data can be compared to ours. It then turns out that, while the pattern of MSD curves matches well between the two systems, the volume fractions of the superimposing curves do not correspond well (see supplementary material [25]).

Reference data are also available for systems of poly(hydroxystearic acid) coated spheres suspended in tetralin/ decalin/carbon tetrachloride [15], cycloheptylbromide/ decalin [9], and cyclohexylbromide/decalin [1,2]. However, these studies address normalized times that are much longer, and in a range that gives only a small overlap with our data (see supplementary material [25]). Also at these longer time scales, the normalized MSD curves of the various systems do not show the overlap expected for ideal hard spheres.

Comparison with literature for (near) HS systems thus leads to two conclusions: (1) the dynamics of our bulk silica suspensions qualitatively resembles that of other (near) hardsphere fluids and (2) the MSDs of several (near) HS systems do not all collapse onto the expected master curve. This suggests that the particle dynamics in near HS fluids is rather 
sensitive to small deviations from ideal HS behavior and underlines the value of MSD measurements in bulk, as a reference for analyzing the dynamics under confinement.

\section{B. Asymmetry between the two confining surfaces}

From the AFM topography images (Fig. 2) it was clear that whereas the cover slip was very smooth, the surface roughness of the glass sphere was certainly not negligible when compared to the size of the silica particles. The layering that we found at the smooth wall and the almost complete absence thereof at the rough wall (Fig. 6) are also in good agreement with expectations for ordering phenomena at smooth and rough walls: in several previous studies (e.g., $[18,20])$ such a behavior has been found. Taking these observations together with the strong effects of wall roughness on local particle dynamics as found in computer simulations $[4,18,20]$, it seems rather likely that the different MSDs found at the two glass surfaces are to be attributed to the differences in the wall roughness.

However, since the mass density of our particles exceeds that of the solvent by $\approx 800 \mathrm{~kg} / \mathrm{m}^{3}$, it cannot be excluded $a$ priori that also gravity could affect the particle dynamics (and hence contribute to the asymmetry between top and bottom surfaces). An estimation of the importance of gravity can be made by comparing the buoyancy force on a single particle to the thermal force $k T / a$ (with $a$ as the particle radius). Expressed as a Peclet number $\mathrm{Pe}=\Delta \rho g a^{4} / k T[41,42]$ we obtain $P e=0.2$. This suggests that the thermal forces are stronger, but also indicates that the effect of gravity should not be completely ignored. Another way of assessing the importance of gravity is to look at the $\varphi(Z)$ profiles for $Z$ $>10 \mu \mathrm{m}$. While the data in Fig. 6 indeed show a decreasing trend, taking this effect of gravity on $\varphi$ into account as in Fig. 5 (open symbols), it came out that a substantial part of the reduction in MSD at the bottom wall (compared to the bulk) cannot be attributed to the volume fraction change. This corroborates once more that the surface effects dominate.

\section{Relative importance of wall and confinement effects}

The availability of different wall surfaces (in contact with the same fluid) together with the ability to control $H$ makes it interesting to compare the effects of the single walls and the confinement distance on the MSD. This is illustrated in Fig. 8, which presents the same data as in Fig. 5, but now as a function of the normalized distance from both walls. Also the magnitude of the bulk MSD at $\varphi=0.33$ (obtained by interpolation) is included. The data at $H=20 \mu \mathrm{m}$ show a large MSD plateau with a magnitude close to that of the bulk system. This means that the effects of the separate walls (i.e., without the influence of a second surface) can be estimated from the difference between the solid and dashed black lines. Clearly, for the smooth wall the reduction in MSD is appreciable and extends over long distance, whereas for the rough wall the effect is small and short ranged. Figure 8 also makes clear that both smooth wall and confinement can cause a major reduction in MSD, and moreover that layering and confinement can also work together: even very close to the

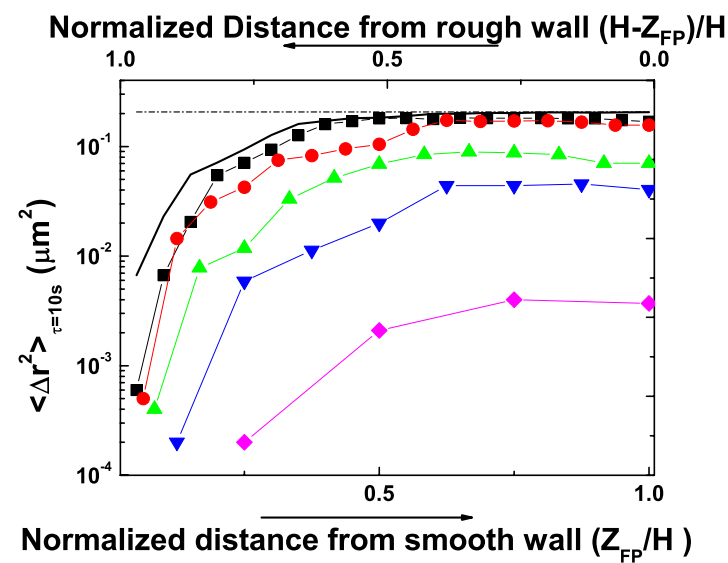

FIG. 8. (Color online) Alternative representation of the data in Fig. 5, to reveal the contributions of layering and confinement on the MSD at $\tau=10 \mathrm{~s}$. The dashed line indicates the MSD of the bulk fluid (at $\varphi=0.33$ ), whereas the solid black line belongs to the layered system (i.e., in the absence of the top surface). Correspondence between $H(\mu \mathrm{m})$ and symbols: $20(\boldsymbol{\square}), 16(\boldsymbol{\bullet}), 12(\boldsymbol{\Delta}), 8(\boldsymbol{\nabla})$, and $4(\diamond)$.

smooth surface, where the MSD was already reduced by two orders of magnitude due to the layering, the confinement is able to further reduce the MSD by more than an order of magnitude. For the rough wall, it is illustrated that the wall effects become negligible compared to the confinement effects, already at relatively large $H$. This corroborates the approach taken in Ref. [2] where the wall was roughened in order to mitigate layering effects. The implication of these findings for practical cases (such as channels in a microfluidic chip or pore channels) is that the overall dynamics in the cavity could be controlled by engineering the roughness of the walls.

\section{CONCLUSIONS AND OUTLOOK}

We investigated the dynamics of dense hard-sphere suspensions under confinement between smooth and rough surfaces. Upon decreasing the gap height $H$, the overall particle dynamics was found to slow down dramatically, in accordance with previous studies. Systematic experiments allowed us to conclude that this slowing down is predominantly a direct effect of confinement and only slightly due to the confinement-induced variations in particle density. Within the gap, local differences in the dynamics were found. Local MSD minima occurring at the walls indicated that besides the confinement effect, wall effects can also contribute to the dynamics. In the case of a smooth wall, interplay between the effects of layering and confinement occurs.

Our finding that volume fraction alone cannot explain the variations in MSD for layered or confined systems confirms that studies of mechanistic details will be needed to achieve a deeper understanding. Finally, we also remark that the dynamics of confined (near) HS suspensions appears to be sensitive to details of the interparticle and the particle-wall interactions. Choosing very similar materials for the particles and walls could help us to better define the systems. Also 
more systematic experiments regarding the wall roughness should be performed.

\section{ACKNOWLEDGMENTS}

We would like to thank Michiel Hermes for fruitful discussions, Sissi de Beer for AFM analysis, Klaas Smit for mechanical assistance, and Mariska van der Weide-Grevelink and Cor Harteveld for technical support. Eric Weeks is acknowledged for providing particle tracking software. We thank the Chemical Sciences Division of the Netherlands Organization for Scientific Research (NWO-CW) for financial support (ECHO grant).
[1] C. R. Nugent et al., Phys. Rev. Lett. 99, 025702 (2007).

[2] P. S. Sarangapani and Y. X. Zhu, Phys. Rev. E 77, 010501 (2008).

[3] G. B. McKenna, J. Phys. IV 10, 53 (2000).

[4] T. Fehr and H. Lowen, Phys. Rev. E 52, 4016 (1995).

[5] P. N. Pusey and W. Vanmegen, Nature (London) 320, 340 (1986).

[6] W. van Megen and P. N. Pusey, Phys. Rev. A 43, 5429 (1991).

[7] P. N. Pusey and W. van Megen, Phys. Rev. Lett. 59, 2083 (1987).

[8] A. Vanveluwen et al., Faraday Discuss. 83, 59 (1987).

[9] E. R. Weeks et al., Science 287, 627 (2000).

[10] W. van Megen, S. M. Underwood, and P. N. Pusey, Phys. Rev. Lett. 67, 1586 (1991).

[11] A. Vanveluwen et al., J. Chem. Phys. 87, 4873 (1987).

[12] A. J. Banchio and G. Nagele, J. Chem. Phys. 128, 104903 (2008).

[13] A. Sierou and J. F. Brady, J. Fluid Mech. 448, 115 (2001).

[14] E. R. Weeks, J. C. Crocker, and D. A. Weitz, J. Phys.: Condens. Matter 19, 205131 (2007).

[15] W. K. Kegel and A. van Blaaderen, Science 287, 290 (2000).

[16] E. R. Weeks and D. A. Weitz, Chem. Phys. 284, 361 (2002).

[17] J. Mittal et al., Phys. Rev. Lett. 100, 145901 (2008).

[18] P. Scheidler, W. Kob, and K. Binder, EPL 59, 701 (2002).

[19] P. A. Thompson, G. S. Grest, and M. O. Robbins, Phys. Rev. Lett. 68, 3448 (1992).

[20] P. Scheidler, W. Kob, and K. Binder, Eur. Phys. J. E 12, 5 (2003).

[21] Z. T. Németh and H. Lowen, Phys. Rev. E 59, 6824 (1999).

[22] R. P. A. Dullens and W. K. Kegel, Phys. Rev. E 71, 011405 (2005).

[23] H. B. Eral et al. (unpublished).

[24] N. A. M. Verhaegh and A. Vanblaaderen, Langmuir 10, 1427
(1994).

[25] See EPAPS Document No. E-PLEEE8-80-006912 for graphs comparing different hard spherelike systems, particle size distributions, and overall volume fractions. For more information on EPAPS, see http://www.aip.org/pubservs/epaps.html.

[26] V. I. Uricanu and M. H. G. Duits, Langmuir 22, 7783 (2006).

[27] W. Schaertl and H. Sillescu, J. Stat. Phys. 77, 1007 (1994).

[28] A. Kasper, E. Bartsch, and H. Sillescu, Langmuir 14, 5004 (1998).

[29] J. C. Crocker and D. G. Grier, J. Colloid Interface Sci. 179, 298 (1996).

[30] M. H. G. Duits et al., Phys. Rev. E 79, 051910 (2009).

[31] T. Savin, P. T. Spicer, and P. S. Doyle, Appl. Phys. Lett. 93, 024102 (2008)

[32] J. P. Gao, W. D. Luedtke, and U. Landman, Phys. Rev. Lett. 79, 705 (1997).

[33] D. H. Van Winkle and C. A. Murray, J. Chem. Phys. 89, 3885 (1988).

[34] C. Murray, MRS Bull. 23, 33 (1998).

[35] T. Savin and P. S. Doyle, Biophys. J. 88, 623 (2005).

[36] J. C. van der Werff and C. G. de Kruif, J. Rheol. 33, 421 (1989).

[37] S. E. Phan, W. B. Russel, Z. Cheng, J. Zhu, P. M. Chaikin, J. H. Dunsmuir, and R. H. Ottewill, Phys. Rev. E 54, 6633 (1996).

[38] T. G. Mason, Rheol. Acta 39, 371 (2000).

[39] T. A. Waigh, Rep. Prog. Phys. 68, 685 (2005).

[40] R. H. Ottewill and N. S. J. Williams, Nature (London) 325, 232 (1987).

[41] J. P. Hoogenboom, P. Vergeer, and A. van Blaaderen, J. Chem. Phys. 119, 3371 (2003).

[42] K. E. Davis, W. B. Russel, and W. J. Glantschnig, Science 245, 507 (1989) 